\title{
Ueber die Anwendung des Wasserstoffs in der
}

\section{Mineralanalyse ;}

\author{
von L. E. Rivot *).
}

Die reducirende Wirkung, welche das trockne Wassersloffgas bei mehr oder weniger erhöhler Temperalur auf mehrere Metalloxyde ausuibt, kann unit grofsem Vortheil zur Trenung dieser Metalloxyde von anderen Basen angewendel werden, auf welche das Wasserstolfgas bei jeder Temperalur ohne Einwirkung ist. Den Versuchen, welche zur Benulzung dieses Vorhaltens schon früher angestellt worden sind, will ich die Resultate mehrerer 'Trennungen und Analysen zufügen, welche nach den gewöhnlich angewendeten Methoden scliwierig auszuführen sind. Ich konnte durch Anwendung des Wasserstoffs mit grofser Genauigkeit das Eisenoxyd von der 'Thonerde, Beryllerde und Zirkonerde, das Eisenoxyd ron dem Chromoxyd, das Zinnoxyd von der Kieselerde trennen.

Trenuung des Eisenoxyds von der Thonerde. - Das in den verschiedenen Lehrbüchern der analylischen Chemie angegebene Verfaluren besteht in der Behandlung des gewogenen Gemenges der beiden Oxyde mit Kali - auf trocknem Wege im Silberliegel oder auf nassem Wege nach Auflösen in einer Säure - , Trennung des Eisenoxyds und Auswaschen desselben mit siedendem Wasser. Dieses Verfahren ist langwierig und of ungenau, wenn das angewendele Kali nicht vollkommen rein ist. Da die Trennung der beiden Oxyde bei der Analyse von Mineralien und von Hültenproducten läufig vorkommt, suchte ich ein schneller ausfựhrbares und sichereres Verfahren. Ich erhielt sehr genaue Resullate, indem ich das gewogene Gemenge von Eisenoxyd und Thonerde, die aus der Lösung in einer Säure

*) Annales de chinie et de physique, 3me série, XXX, 188. 
durch Ammoniak niedergeschlagen waren, bei Rolhglühhitze der reducirenden Wirkung von Wasserstoff aussetzte, in einer Wasserstoflatmosphäre erkalten liefs, und das entstandene Gemenge von 'Thonerde und metallischem Eisen mit sehr verdünnter Salpelersäure in der Kälte behandelte, wo sich das Eisen leicht auflöst, während die slark geglühte Thonerde nicht angegriffen wird.

Die Angabe von wenig Einzelnheiten wird hinreichen, dieses Verfahren deutlich zu machen.

Das Eisenoxyd und die Thonerde werden zusammen durch Ammoniak gefällt; der Niederschlag wirl getrocknet und von dem Filter getrennt; das Filter wird verbrannt und die Asche den beiden Oxydhydraten rugefügt; das Ganze wird in einem Platintiegel bei guter Rolhgliihhilze geglüht. Das Gemenge der beiden 0xyde wird fein zerrieben und genau gewogen, dann in einem gleichfalls gewogenen Porcellanschiffchen in ein Porcellanrohr gebracht, welches in einem Ofen horizontal liegt. An dem einen Ende des Rohres ist ein dünn ausgezogenes Glasrohr angebracht, durch das andere läfst man einen langsamen Strom von Wasserstoflgas eintreten, welches durch Leiten über Chlorcalcium und durch Schwefelsäurehydrat getrocknet ist.

Wenn die Luft aus dem Apparat vollstândig ausgelrieben ist, so erhilzt man allıälig das Porcellanrohr bis zum Rothglühen, und erhält diese Temperalur so lange, als man Wasser an den Wandungen des erwähnten Glasrohrs sich absetzen sieht. Bei keinem meiner Versuche brauchte ich länger als eine Stunde zu erhilzen, um die Reduclion zu Ende zı führen.

Man läfst dann das Porcellanrohr erkalten, indem man fortwährend Wasserstoff hindurchleitet. Wenn das Porcellanrohr erkaltet ist, nimmt man das Schiffchen heraus und wiegt es; der Gewichtsverlust giebt den Sauerstoffgehalt des Eisenoxyds an unl erlanbl, die Quantilat desselben in dem Gemenge zu Irrechncm. Doch ist es nicht rallsam, sich mil dieser Bestim- 
mung zu begnügen; wenn eine grofse Menge von Thonerde vorhanden ist und man nicht die Vorsicht brauchte, das Wasserstoffgas sehr langsam zutreten zu lassen, so kann etwas Thonerde durch das Wasserstoffgas mit fortgerissen worden seyn, und der Gewichlsverlust ergäbe also einen zu grofsen Gehalt an Eisenoxyd.

Das Gemenge von metallischem Eisen und Thonerde wird während elwa 24 Stunden und in der Kälte mit selır verdünnter Salpetersäure digerirt. Man mufs die gewöhnliche reiıe Säure anwenden, die wenigstens mit ilrrem 30 fachen Volum Wasser verdünnt ist, oder besser noch verdünntere Säure, oder reines Wasser und wiederholt kleine Mengen Salpetersäure zusetzen, so dals eine schwache Entwicklung von Wasserstoffgas unlerhalten wird; bei diesem Verfahren ist man sicher, alles Eisen aufzulösen, ohne die Thonerde anzugreifen. Die Auflösung des Eisens ist vollständig, wenn die Thonerde fast weifs geworden ist.

Man scheidet die ungelöste Thonerde durch Filtralion. Aus der salpetersauren Lösung, die man vorher erhilzt, um das Eisen in Oxyd überzuführen, fällt man das Eisenoxyd mit Ammoniak.

Man kann auf diese Weise die Thonerde nnd das Eisenoxyd jedes für sich bestimmen.

Wenn die Menge der Thonerde nur gering ist; ergiebt schon der Gewichtsverlust bei dern Glühen des Gemenges in Wasserstoffgas, als der Ausdruck für den Sauerstoffgehalt des Eisenoxyds, ein sehr genaues Resultat.

Ich habe die folgenden genau abgewogenen Gemenge dem Versuch unterworfen :

$$
\text { I. Il. } 111 \text {. }
$$

$\begin{array}{llll}\text { Thonerde } & \mathbf{0 , 5 0 0} \mathbf{G r m} . & \mathbf{0 , 1 5 2} & \mathbf{0 , 0 5 3} \\ \text { Eisenoxyd } & \mathbf{0 , 5 0 0} \text {, } & \mathbf{0 , 4 2 7} & \mathbf{0 , 5 2 6}\end{array}$

und folgende Zahlen erhalten :

$\begin{array}{lllll}\text { Gewichtsverlust im Wasserstoff } & 0,156 & \# & 0,132 & 0,1616 \\ \text { entsprechend Eisenoxyd } . \cdots & 0,510 & 0,431 & 0,527 .\end{array}$


Bei dem Wägen des Eisenoxyds und der Thonerde, welehe auf dic eben angegebéne Weise mittelst schwacher Salpetersäurc getrennt waren, erhielt ich :

$\begin{array}{llll}\text { Thonerde } & \mathbf{0 , 4 9 2} & 0,148 & 0,052 \\ \text { Eisenoxyd } & \mathbf{0 , 4 9 8} & \mathbf{0 , 4 2 8} & \mathbf{0 , 5 2 4}\end{array}$

Es gelst aus diesen Zahlen hervor, dals die Trennung der beiden Oxyde durch das angegebene Verfahren eine sehr scharle ist ; dafs die beiden Oxyde genau bestimml werden, indem man nur das Eisenoxyd direct bestimmit und die Thonerde aus dem Verlúste berechnel; und endlich, dafs der Gewichisverlust im Wasserstoff den Gehalt an Eisenoxyd in Folge des Fortreifsens durch den Gasstrom etwas zu hoch ergicbt, wenn die Menge von Thonerde elwas bedeutender ist, und dafs er im Gegentheil ein sehr genaues Resultat ergiebt, wenn die Menge der Thonerde nur gering ist.

Ich muls hier noch bemerken, dals, wenn man Wasserstoff über ein mechanisches Gemenge von Thonerde und Eisenoxyd leitel, anstall äber ein Gemenge der beiden $0 x y d e$, wie es durch Ammoniak niedergeschlagen wird, dic 'Thonerde vicl leichler durch den Gasstrom forlgerissen wird.

Um aus dem Gewichtsverlust in Wasserstolf die entsprechende Menge Eisenoxyd zu berechnen, habe ich für das Atomgewicht des Eisens die früher angenommene Zahl 339 benutzt. Diese Zahl ergaben mir zwei Versuche, wo mit der grölsten Sorgfalt 1 Gramm reines Eisenoxyd in Wassersloff reducirl wurde; ich erhielt 0,6931 und 0,6935 Gramm metallisches Eisen, und das Miltel dieser sich sehr nahe kommenden Zahlen führt fast genau zu der Zahl 339 für das Atomgewicht des Eisens.

Trennung des Eisenoxyds und der Zirkonerde. - Die Trennung dieser beiden Basen durch die Reduclion des Eisenoxyds miltelst Wasserstoff geht mit derselben Leichtigkeit und Genauigkeit vor sich, wie die des Eisenoxyds und der Thonerde. Das durch Wägung der Zirkonerde erhaltene Resultat ist genauer, weil diese Erde specifisch schwerer ist als die Thonerde, und defshalb nicht bemerkbar durch den Gasstrom forlgerissen wird. 
Man kann auch zur Auflösung des reducirten Eisens weniger verdünnte Salpelersäure anwenden, weil die geglühte Zirkonerde sich nicht in Säuren auflöst.

Ich unterwarf dem Versuch das Gernenge :

$$
\begin{array}{ll}
\text { Eisenoxyd } & 0,660 \mathrm{Grm} . \\
\text { Zirkonerde } & 0,377,
\end{array}
$$

und erhielt folgende Resultate :

$$
\begin{array}{ll}
\text { Gewichtsverlust im Wasserstoff } & 0,205 \\
\text { entsprechend Eisenoxyd - . } & 0,667,
\end{array}
$$

Durch Wägung der Zirkonerde und des Eisenoxyds erhielt ich :

$$
\begin{array}{ll}
\text { Eisenoxyd } & 0,668 \mathrm{Grm} . \\
\text { Zirkonerde } & \mathbf{0 , 3 7 5},
\end{array}
$$

Diese Zahlen beweisen, dals die Trennung der beiden 0xyde nacb dem vorgeschlagenen Verfahren sehr genau ist, und dafs man die Zusammensetzung des Gemenges aus dem Gewichtsverlust in Wasserstoff, als dem Ausdruck des Sauerstoffgehalts des Eisenoxyds, berechnen kann.

Diese Art der Trennung ist rascher und genauer als die bis jetzt vorgeschlagenen andern Melhoden.

Trennung des Eisenoxyds und der Beryllerde. - Diese Trennung läfst sich, mit denselben.Vorsichtsmafsregeln wie bei der Trennung des Eisenoxyds und der Thonerde, ebenso genau ausführen. Die Beryllerde kann, wie die Thonerde, durch den Gasstrom fortgerissen werden, wenn sie in beträchllicher Menge vorhanden ist, und in diesem Falle darf man also nie die $\mathbf{Z u}$ sammenselzung des Gemenges nur aus dem Gewichtsverlust in Wasserstoff ableiten. Man mufs das reducirte Eisen in verdünnter Salpelersäure in der Källe auflösen; die Säure darf nicht weniger als 30 Theile Wasser auf 1 Theil gewöhnliche reine Salpetersäure enthalten.

Ich habe dem Versuch folgendes Gemenge unterworfen :

$$
\begin{array}{ll}
\text { Eisenoxyd } & 0,815 \text { Grm. } \\
\text { Beryllerde } & 0,399 \text {, }
\end{array}
$$

und folgende Zahlen erhallen : 
Gewichtsverlust im Wasserstoff $\quad 0,249$ Gr'm.

entsprechend Eisenoxyd . - 0,812 "

Nuch Behandlung des Gemenges von reducirtem Eisen und Beryllerde mit verdiinnter Salpetersäure fand ich :

$\begin{array}{ll}\text { Eisenoxyd } & 0,816 \text { Grm. } \\ \text { Beryllerde } & 0,397,\end{array}$

Das angegebene Verfahren eignet sich somil sehr gut zur Trennung des Eisenoxyds und der Beryllerde.

Ich habe keine Versuche angestellt hinsichtlich der Trennung des Eisenoxyds von der Yltererde und der Thorerde.

Die reducirende Einwirkung des $W$ asserstoffs auf die Oxyde von Kobalt und Nickel scheint gleichfalls einer nützlichen Anwendung zur 'Trennung dieser Oxyde von der Thonerde fühig zu seyn. Wenn man aus einer Auflösung, welche die Oxyde von Kobalt und Nickel neben Thonerde enthält, die letzlere mit Ammoniak ausfällt, so enthält der Niederschlag eine gewisse Menge der Oxyde von Kobalt und Nickel. Man kann die Thonerde sehr rein erlualten, indem man den mit Ammoniak erhaltenen Niederschlag glüht, mit trocknem Wassersloffgas und dann, wie das Gemenge von Eisenoxyd und Thonerde, mit verdünnter Salpetersäure in der Kälte behandelt, wo nur das Kobalt und das Nickel aufgelöst werden.

Trenung des Zinnoxyds und der Kieselerde. - Die reducirende Einwirkung des Wasserstoffs auf das Zinnoxyd kann mil Vortheil zur scharfen Trennung dieses Oxyds von der Kieselerde benutzt werden.

Ich verfahre in folgender Weise: Das stark geglïhte und gewogene Gemenge von Zinnoxyd und Kieselerde wird in einem gleichfalls gewogenen Porcellanschiffchen in ein Porcellanrohr gebracht, wie bei den im Vorhergehenden besprochenen Versuchen. Nan lälst das Wasserstoflgas sehr langsam zutreten; damil keine Kieselerde durch den Gasstrom forlgerissen werde, und erhitzl nur zum dunkeln Rolhglihhen. Die Reduction des 
Zinnoxyds geht rasch vor sich. Nach dem Erkalten in Wasserstoff hat dus Gemenge von reducirtem Zinn und Kieselerde die Form eines grauen Pulvers ohne metallische Kügelchen, aufser wenn die Temperalur zu hoch gesteigert wurde oder das Zinnoxyd nicht ganz rein war. Man wägt; der Gewichssverlust giebt den Sauersloff des Zinnoxyds und läfst die Zusammenselzung des Gemenges annähernd berechnen. Da indessen eine kleine Menge Kieselerde durch den Gasslrom fortgerissen worden seyn kann, ist es besser, das Zinn in Salpetersalzsäure aufzulösen, die ungelöst bleibende Kieselerde zu wägen, und in dem Falle, dafs ihr Gewicht nicht genau dem aus dem Gewichtsverlust sich berechnenden entspricht, das in der salpetersalzsauren Flüssigkeit enthaltene Zinnoxyd zu bestimmen. Das Verfahren, welches sich mir für diese Bestimmung am besten bewährte, ist folgendes : ich sällige die saure Flüssigkeil mit Ammoniak, selze hinlänglich viel Schwefelammonium zu, um das Zinn aufzulösen, und zersetze das Schwefelsalz des Zinns mittelst Salzsäure, wo das Zinn als Schwefelzinn erhalten wird. Dieses wird nach gehörigem Auswaschen mit Vorsichl geröstel und in Zinnoxyd verwandelt.

Ich unterwarf dem Versuche die Gemenge :

$$
\begin{array}{lll}
\text { Zinnoxyd } & \mathbf{0 , 3 1 0} \mathrm{Grm} . & \mathbf{0 , 5 0 0} \\
\text { Kieselerde } & \mathbf{0 , 6 4 4}, & \mathbf{0 , 5 0 0} .
\end{array}
$$

Ich erhielt :

$$
\text { Gewichtsverlust im Wasserstoff } 0,069,0,106 \text {, }
$$

\begin{tabular}{|c|c|}
\hline & 0,324 Grm. 0,509 \\
\hline esel & 0,630 \\
\hline
\end{tabular}

woraus sich die Zusammenselzung der Gemenge berechnel :

Die Menge des Zinnoxyds wäre hiernach erheblich zu grofs gefunden worden. Durch Behandlung mit Salpetersalzsäure und Bestimmung des Zinns nach der angegebenen Methode erhielt ich :

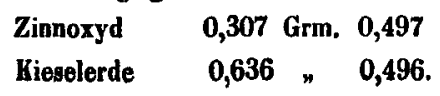


Diese Zahlen beweisen, dals der Fehler, welcher bei Betracilung des Gewichlsverlusis im Wasserstoff als Sauerstoff des Zimnoxyds begangen war, auf dem Fortreifsen einer kleinen Menge Kiesclerde durch den Gasstrom beruhte. Nur in dem Fall, wenn die Menge der Kieselerde sehr gering ist, kann man dieses Fortreifsen durch den Gasstrom vernachlässigen und den Gewichtsverlust als den Sauerstoff des Zinnoxyds betrachten. Wenn dic Menge der Kieselerde etwas beträchtlich ist, mufs man immer das Zinn direct bestimmen und die Kieselerde aus dem Verlust berechnen.

Analyse zinnhaltiger Erze. - Die zinnoxydlialtigen Erze können mit Hülfe des trocknen Wasserstoffgases sehr schnell analysirt werden. Wenn man das fein zerriebene Mineral in einem Strome von trocknem Wasserstoffgas allmälig bis zum Rothglühen erhilzt, so werden das Eisenoxyd und das Zinnoxyd vollständig reducirt. Die zur Reduction nollwwendige Zeit ist elwa anderthalb Stunden für 1 bis 2 Gramm Erz. Man läfst im Wasserstoff erkalten und wägt; der Gewichtsverlust giebt die Menge des mit dem Eisen und dem Zinn verbunden gewesenen Sauerstoffs. Durch Behandlung des Rückstands mit Salpetersalzsäure löst man das Eisen und das Zinn auf; die Gangart des Erzes bleibt ungelöst zurïck und kann nach den gewölnlichen Melhoden analysirt werden *). Zu der Auflösung des

*) In dem Falle, dafs die Gangart ein Gemenge von Quarz und Flufsspath ist, lkann man sie schnell und mit hinreichender Genauigkeit auf folgende Weise analysiren. Das fein zerriebene und gewogene Gemenge von Quarz und Flufsspath wird mit dem achtfachen Gewicht kohlensaurem Natron-Kali im Platintiegel geschmolzen. Die Masse wird während einer Stunde in Schmelzen erhalten und während dieser Zeit mit einem Platinspiegel umgerührt; man mufs aufserdem die gewöhnlichen Vorsichtsmafsregeln beobachten, un ein zu starkes, durch die Kohlensäureentwicklung aus den kohlensauren Alkalien veranlafstes Schäumen zn vermeiden. Die geschmolzene Masse wird auf ein Platinblech zu einer dünnen Platte ausgegossen, 
Eisens und des Zinns in Salpetersalzsäure setzı nun Ammoniak behufs der Sălligung der Säuren, und einen grofsen Ueberschuls von Schweferammonium zur Lösung des Schwefelzinns. Man lälst während etwa 12 Stunden digeriren, und filtrirt alsdann. Das Schwefeleisen wird mil schwefelammonium-haltigem Wasscr wohl gewaschen und nach dem bekannten Verfuhren in Eisenoxyd verwandelt. Das Schwefelzinn wird aus seiner Lösung in Sclıwefelammonium durch Essigsäure niedergeschlagen, und durch Rösten mit den gehörigen Vorsichtsmafsregeln in Zinnoxyd verwandelt.

Die Gewichle des Eisenoxyds und des Zinnoxyds ergeben die Gewichte des Eisens und des Zinns, während der Verlust

dann mit Wasser digerirt. Ohne diese Vorsicht, die Masse in Form einer dünnen Platte zu bringen, geht die Einwirkung des Wassers nur langsam vor sich. Durch die Einwirkung der kohlensaureu Alkalien entstehen kieselsaure Alkalien, Fluor - Alkalimetalle und kohlensaurer Kalk; die geschmolzene Masse enthält aufserdem den Ueberschufs der angewendeten kohlensauren Alkalien. Das Wasser löst diesen Ueberschufs, die kieselsauren Alkalien und die FluorAlkalimetalle auf, und läfst den durch Zersetzung des Flufaspaths entstandenen kohlensauren Kalk zurück. Dieser kohlensaure Kalk wird mit heifsem Wasser gut ausgewaschen, bei dunkler Rothglühhitze geglüht und gewogen. Aus seinem Gewicht berechnet man den Gehalt des Gemenges an Flufsspath. Zur Controle kann man die in der alkalischen Lösung enthaltene Kieselerde bestimmen.

Ich anterwarf dem Versuch die Gemenge :

$\begin{array}{llll}\text { Flufsspath } & 0,65 \mathrm{Grm} .0,50 & 0,25 \\ \text { Quarz } & 0,35,0,50 & 0,75 .\end{array}$

lch erhielt folgende Zahlen :

$\begin{array}{llll}\text { Kohlensauren Kalk } & 0,817, & 0,636 & 0,318 \\ \text { entsprechend Flufsspath } & 0,642, & 0,494 & 0,247 .\end{array}$

Diese Resultate zeigen eine Genauigkeit, welche für die Analyse hinreichend ist, und namentlich um den zur metallurgischen Behandlung der Erze nöthigen Zuschlag zu berechnen.

Ilerr Pach e, früherer Zögling der École des Mines, hat dasselbe Verfahren versucht, und ist zu gleichermafsen befriedigenden Resultaten gelangt. 
im Wasserstoff dic Menge des mil diesen Melallen verbundenen Sauerstoffs ergiebt und auf ihren 0xydationsgrad schliefsen läfst.

Ich will hier die Zahlen mittheilen, welche ich bei der Analyse des Zinnschlichs von Piriac erhalten habc. Dieser Schlich enthält Zinnoxyd in kleinen Körnern, elwas Eisenoxydul, einige Granaten und Quarz.

Gewicht des Schlichs vor dem Glühen in Wasserstuff 1,1795 Gim. Gewichtsverlust bei dem Glühen in Wasserstoff . . 0,1235 , Gewicht der in Salpetersalzsäure unlöslichen und zinn-

freien Gangart . . . . . . . . . . . . 0,5650 , In der Salpetersalzsäure gelöstes Eisen . . . . 0,1310 , " " "Zinn . . . . . . 0,3624,

Diese Zahlen geben für 1 Gewichlstheil Zinnschlich die Zusammensetzung :

\begin{tabular}{|c|c|c|}
\hline Eisen . . & . . & 0,1110 \\
\hline Zinn & & 0,3081 \\
\hline Verbundener & Sauerstolf & 0,1047 \\
\hline Gangart & . & 0,4790 \\
\hline
\end{tabular}

Ich inache darauf aufmerksam, dafs das Eisen, das Ziun und der Sauerstoff unter sich nahezu in den Aequivalentsgewichtverhältnissen $4: 5: 12$ stehen. Ich will keine Hypothese uber die chemische Formel des Zinnsleins hieraus ableiten; ich hoffe nächstens mehrere gut krysiallisirte Exemplare von Zinnstein zu analysiren und die Art zu bestimmen, wie das Eisen und das Zinn mit dem Sauerstoff verbunden sind. Ich habe die vorstehende Analyse nur angeführt, um zu zeigen, wie schnell man das Eisen und das Zinn in einem Zinnerz bestimmen kann, wenn man damit beginnt, das fein gepulverte Erz mit Wasserstoff $z u$ behandeln.

Trennung des Eisenoxyds und des Chromoxyds. - Das zur Trennung des Eisenoxyds und des Chromoxyds gewöhnlich angewendete Verfahren ist folgendes : Der salzsauren Auflösung beider $0 x y d e$ setzt man soviel Weinsäure zu, dals bei Sätligung 
der Säuren mit Ammoniak sich kein Niederschlag bildet; dann schlägt man mit Schwefelammonium das Eisen als Schwrfeleisen nieder; das Chromoxyd bleibt aufgelöst; um das Chrom zu bestimınen, mufs man die weinsäurehaltige Flüssigkeit zur 'frockne abdampfen, diese Säure durch Rösten zerstören und endlich das Chrom als Chromoxyd $\mathrm{Cr}_{2} \mathrm{O}_{3}$ wägen.

Dieses Verfahren ist langwierig und führt nicht zu einer ganz genauen Trennung. Die folgende Melhode scheint mir schneller zum Zicle zu führen und ganz genau zu seyn. Sie gründet sich darauf, dafs der Wasserstoff bei Rothglühhilze das Eisenoxyd leicht reducirl und auf das Chromoxyd keine Einwirkung ausübt.

Das Gemenge beider Oxyde, wie es durch die Fällung mit Ammoniak crhallen wird, wird geglüht und gewogen, und so behandell, wie es für das Genenge von 'Thonerde und Eisenoxyd oben angegeben wurde. Nach dem Erkalten im Wassersloff eutliält die Masse melallisches Eisen und Chromoxyd. Der Gewichtsverlus! kommt ausscliliefslich auf Rechnung des mil deın Eisen verbunden gewesenen Sauerstofls, und gestaltel eine selir genaue Bestimnung des Eisenoxyds und somit der Zusammensetzung des Gemenges. Zur Controlle kann man die Masse nit verdiinnter und kalter Salpetersäure belıandeln, wo sich das Eisen allein auflöst; man kann das ungelöst zurückbleibende Chromoxyd wägen, und das Eisenoxyd aus der salpetersauren Lösung mil Ammoniak ausfällen; beide Bestimmungen geben sehr genaue Resultate.

Ich unterwarf dem Versuch folgende Gemonge :

$\begin{array}{llll}\text { Chromoxyd . . . . } & \mathbf{0 , 3 0} \text { Grm. } & \mathbf{0 , 7 6 1} \\ \text { Eisenoxyd . . . . . } & \mathbf{0 , 7 0} " & \mathbf{0 , 7 4 9} \\ \text { Verlust im Wasserstoff } & \mathbf{0 , 2 1 3} " & \mathbf{0 , 2 3 0} \\ \text { entsprechend Eisenoxyd } & \mathbf{0 , 7 0 5}, & \mathbf{0 , 7 4 9 8} .\end{array}$

Bei nachheriger Behandlung mit verdünnter Salpetersäure, Wägen des ungelöst bleibenden Chromoxyds und Niederschlagen des Eisenoxyds mit Ammoniak erhielt ich : 


$\begin{array}{llll}\text { Chromoxyd } & 0,30 & \text { Grm. } & 0,761 \\ \text { Eisenoxyd } & 0,704 \quad \cdots & 0,750 .\end{array}$

Diese Zahlen reichen wohl hin zum Beweise, dafs die Trennung und die Bestimınung der beiden Oxyde vollkommen genau sind.

Analyse des Chromeisensteins. - Der Chromeisenstein kann nach folgendein Verfahren mit grofser Genauigkeit analysirt werden. Das fein zerriebene Mineral wird in einem Porcellanschiffchen in eine Porcellanrühre gebracht, welche horizontal in einem Ofen liegt, und bei starker Rolhglühhitze während vier Slunden der Einwirkung eines Stroms von trocknem Wassersloffgas ausgesetzt. Alles Eisenoxyd wird reducirt *). Der Gewichtsverlust giebt die Menge des mit dem Eisen verbunden gewesenen Sauerstoffs an.

Die mit Wasserstoff behandelte Masse wird während vier und zwanzig Stunden mit verdünnter Salpetersäure bei gelinder Wärme digerirt; die Säure löst das Eisen und eine Spur von Kalk auf, ist aber ohne Einwirkung auf das Chromoxyd, die Kieselerde, die Thonerde und selbst den Kalk.

Das Eisenoxyd wird mit Ammoniak aus der salpetersauren Lösung niedergeschlagen. Der in der Säure nicht gelöste Theil wird im Silberliegel mit einem Gemenge von 1 Theil Salpeter, 3 kohlensaures Natron und 3 Aetzkali geschmolzen. Man erhält das wohlgeschmolzene Gemenge wäbrend ciner Slunde bei anfangender Rothglühhitze.

Die erkaltete Masse wird mit heifsem Wasser behandelt, worin sie sich schnell und ohne Rückstand löst; die Flüssigkeit ist gelb gefärbt. Man selø! zu der wässerigen Lösung soviel

*) Mehrere Chemiker haben behauptet, dafs der Chromeisenstein nur theilweise durch Wasserstofr angegriffen werde; die Reduction geht schwierig und langsam vor sich, aher in vier Stunden kann man alles Eisen reduciren, vorausgesetzt, dafs das Mineral fein gepulvert war. 
Salzsäure, bis deutliche saure Reaction vorhanden ist, und damp ft sie dann mil Alkohol zur 'Trockne ab. Den Rückstand behandelt man mit Salzsäure; dic Kieselerde bleibt ungelöst. Die saure Lösung enthält die Chlorverbindungen von Chrom, Aluminium und Calcium. Man filtrirt zur Trennung der Kieselerde und fältt nit Ammoniak; der Niederschlag entlualt Chromoxyd, Thonerde Imd elwas Kalk. Man sammelt den Niederschlag auf einem Filter, und trocknet und glüht ilın mit den gewöhnlichen Vorsichtsmafsregeln, unter Beachlung des Umstands, dafs man ihn gegen das Ende des Erhilzens lebhaft roth glühen lassen muls. Um den heigemengten Kalk zu trennen, behandelt man den stark geglühten Niederschlag in der Källe mil schwacher Essigsäure; man vereinigt die essigsäurehallige Flüssigkeit mit der ammoniakalischen Lösung, fällt den Kalk mit oxalsaurem Aınmoniak und untersucht, ob die Flüssigkeit Magnesia enthält.

Man wägt das von neuem geglühte Gemenge von Chromoxyd und Thonerde, und behandelt dann diefs Gemenge mil Salzsäure, welche nur die Thonerde auflöst; man wägt das ungelöst geblicbene Chromoxyd; man fält die Thonerde mit Ammoniak. In dem Falle, dafs der Thonerde noch elwas durch die Salzsäure gelöstes Chromoxyd beigemischt wäre, mülste man die Trennung mittelst Kali bewerkstelligen.

Ich kommle nach diesem Verfahren nur Eine Varieläl von Chromeisenstein, den von Ballimore, untersuchen. Das Mineral bildel kryslallinische, sehr glänzende Körner; eine geringe Menge derselben wird durch den Magnet angezogen. Ich Irennte diese vor Ausführung der Analyse; es war Titaneisen.

Die Analyse gab mir folgende Zahlen : Gewicht des Chromeisensteins . . . 1,581 Grm.

Gewichtsverlust im Wasserstoff . . . 0,144 "

Eisenoxyd, aus der salpetersauren Lösung durch Ammoniak niedergeschlagen . 0,475 "

Die salpelersaure Lösung enthielt aufserdem eine Spur Kalk. Es ist zu beachlen, dals der Gewichtsverlust 0,144 ciner Menge 
224 Rivot, über die Anwendung des Wasserstoffs etc.

Bisendxyd $=0,4694$ entspriclit, beinahe $=0,475$, wie. sie durch Fällung mit Ammoniak gefunden wurde; dieses beweist, dafs das Eisen in diesem Mineral als Eisenoxyd enlhalten ist.

$$
\text { Kieselerde oder Titansüure } \quad 0,035 \text { Grm. }
$$

Jclr konnte bei dieser, geringen Menge die Titansiure nicht mit Gewifsheit unterscheiden.

$\begin{array}{ll}\text { Chromoxyd } & 1,002 \text { Grm. } \\ \text { Thonerde } & 0,030 \\ \text { Kalk } & 0,032,\end{array}$

Das Mineral entlält keine Magnesia.

Diese Zahlen füliren zu folgender Zusammensetzung für 1 Theil des Minerals :

\begin{tabular}{|c|c|c|}
\hline Eisenoxyd & . . . . & 0,3004 \\
\hline Thonerde & . & 0,0196 \\
\hline Kalk . & . & 0,0202 \\
\hline Kieselerde, & , Titansiure? & 0,0221 \\
\hline Chromoxyd & . & 0,6337 \\
\hline
\end{tabular}

Ich will hinsichllich dieser Zusammenselzung nur eine einzige Bemerkung machen : Der Sauerstoffgehalt des Eisenoxyds und der Thonerde ist nahezu die Hälfte des Sauerstoffgehalts des Chromoxyds. Ich denke nächstens andere Varielälen des Chromeisensteins zu untersuchen und zu prüfen, ob dieses Verhältuifs constant ist.

Mehrere Mineralien künnen leicht mit Hülfe des trocknen Wasserstoffgases zerlegt werden. Ich hoffe bald die vorstehenden Beoblachlungen vervollständigen zu können, und mit einiger Sicherheil die chemische Zusammenselzung einer gewissen Zahl von Substanzen zu bestimmen, die durch Säuren schwierig oder gar nicht angegriffen werden. 